\title{
Glutamine synthetase encoded by $g \ln A-1$ is necessary for cell wall resistance and pathogenicity of Mycobacterium bovis
}

\begin{abstract}
Correspondence
Nirupama Banerjee

nirupama@icgeb.res.in
\end{abstract}

Received 16 July 2010

Revised 17 August 2010

Accepted 17 August 2010

\author{
Harish Chandra, ${ }^{1}$ Seemi Farhat Basir, ${ }^{2}$ Manish Gupta ${ }^{1}$ \\ and Nirupama Banerjee ${ }^{1}$
}

\author{
${ }^{1}$ International Centre for Genetic Engineering and Biotechnology, Aruna Asaf Ali Marg, New Delhi \\ 110067, India \\ ${ }^{2}$ Department of Biosciences, Jamia Millia Islamia, New Delhi 110025, India
}

\begin{abstract}
Pathogenic strains of mycobacteria produce copious amounts of glutamine synthetase (GS) in the culture medium. The enzyme activity is linked to synthesis of poly- $\alpha$-L-glutamine (PLG) in the cell walls. This study describes a glnA-1 mutant of Mycobacterium bovis that produces reduced levels of GS. The mutant was able to grow in enriched $7 \mathrm{H} 9$ medium without glutamine supplementation. The $g \ln A-1$ strain contained no detectable PLG in the cell walls and showed marked sensitivity to different chemical and physical stresses such as lysozyme, SDS and sonication. The sensitivity of the mutant to two antitubercular drugs, rifampicin and D-cycloserine, was also increased. The glnA-1 strain infected THP-1 cells with reduced efficiency and was also attenuated for growth in macrophages. A Mycobacterium smegmatis strain containing the $M$. bovis $g \ln A-1$ gene survived longer in THP-1 cells than the wild-type strain and also produced cell wall-associated PLG. The $M$. bovis mutant was not able to replicate in the organs of BALB/c mice and was cleared within 46 weeks of infection. Disruption of the $g \ln A-1$ gene adversely affected biofilm formation on polystyrene surfaces. The results of this study demonstrate that the absence of $g \ln A-1$ not only attenuates the pathogen but also affects cell surface properties by altering the cell wall chemistry of the organism via the synthesis of PLG; this may be a target for drug development.
\end{abstract}

\section{INTRODUCTION}

Tuberculosis is a major cause of mortality and morbidity around the world. The disease is caused by different species of mycobacteria, transmitted primarily through the respiratory route into the lungs and spread to other organs through the haematogenous system (Flynn \& Chan, 2001). Mycobacterium tuberculosis and Mycobacterium bovis are the two major species that infect humans and animals, inflicting huge losses in terms of human health and the economy. Therapeutic intervention is achieved through a multiple drug regimen that involves a combination of antibiotics. However, the emergence of multidrug-resistant strains has prompted worldwide efforts to develop innovative vaccine strategies and novel drug targets for eradication of the disease.

Nitrogen metabolism plays a central role in bacterial physiology. It is linked to other metabolic networks

Abbreviations: GS, glutamine synthetase; PLG, poly- $\alpha$-L-glutamine; REMA, resazurin microplate assay.

A supplementary figure, showing the construction of the $M$. bovis $g \ln A-1$ mutant, and two supplementary tables, showing bacterial strains and plasmids, and PCR primers, used in this study, are available with the online version of this paper. through the key molecules glutamine and glutamate. Glutamine acts as a nitrogen donor for many reactions in the cells, and is primarily synthesized from L-glutamate, ammonia and ATP by the enzyme glutamine synthetase (GS) (Reitzer, 1996; Fisher, 1999; Merrick \& Edwards, 1995). In mycobacteria, GS and glutamate synthetase are the sole means of ammonia assimilation under nitrogenlimiting conditions.

Several mycobacterial species are known to possess multiple genetic loci that encode GS (Harper et al., 2008; Amon et al., 2009). Both M. bovis and M. tuberculosis have four different genes encoding GS (Cole et al., 1998; Garnier et al., 2003). In M. tuberculosis, the $g \ln A-1$ gene encodes a class I GS (GSI) enzyme that is released into the culture medium and plays a crucial role in pathogenicity (Harth et al., 1994), while the other three $g \ln A$ genes $(g \ln A-2$, $g \ln A-3$ and $g \ln A-4)$ encode cytoplasmic proteins. All GSs are multimeric enzymes and catalyse synthesis of L-glutamine, while GlnA2 catalyses the synthesis of D-glutamine and isoglutamine. Only $g \ln A-1$ has been shown to be essential for M. tuberculosis growth (Harth et al., 2005). Disruption of $g \ln A-1$ in $M$. tuberculosis results in glutamine auxotrophy, indicating its role in the synthesis of glutamine for the 
bacillus (Tullius et al., 2003). The genome of the saprophyte Mycobacterium smegmatis $\mathrm{mc}^{2} 155$ also contains homologues of $g \ln A-1-4$, in addition to several sequences that encode GSs similar to those present in soil-dwelling bacteria but absent from the mycobacterial genome (Amon et al., 2009).

GS is implicated in providing glutamine for the synthesis of the poly- $\alpha$-L-glutamine (PLG) layer associated with the cell wall of pathogenic mycobacteria, accounting for as much as $10 \%$ of their weight (Wietzerbin et al., 1975; Hirschfield et al., 1990). It is absent in saprophytic and other nonpathogenic strains (Wietzerbin et al., 1975). Treatment of M. tuberculosis with an inhibitor of GS, L-methionine-Ssulfoxamine, or with antisense oligonucleotides to $g \ln A-1$ mRNA, has been shown to inhibit PLG formation in the cell wall (Harth et al., 2000). However, there is no direct evidence to demonstrate the involvement of $g \ln A-1$ in the synthesis of PLG and its role in fortifying the cell envelope of the pathogen.

$M$. bovis contains an array of genes involved in the uptake and synthesis of glutamine identical to that of $M$. tuberculosis; however, the role of different proteins has not been studied in this pathogen. Since $g \ln A-1$ is necessary for synthesis of glutamine, a key cell envelope constituent, its role in modulating cell wall chemistry needed to be investigated as a potential drug target against mycobacteria. This study is focused on examining the role of GS in the stress tolerance and cell wall resistance of M. bovis.

\section{METHODS}

Bacterial strains and plasmids. Bacterial strains and plasmids are listed in Supplementary Table S1. M. bovis strains were grown on Middlebrook $7 \mathrm{H} 10$ or $7 \mathrm{H} 11$ agar (Difco) containing $10 \%(\mathrm{v} / \mathrm{v})$ oleic acid, albumin, dextrose and catalase (OADC) (Becton Dickinson), $0.5 \%(\mathrm{v} / \mathrm{v})$ glycerol and $0.05 \%(\mathrm{v} / \mathrm{v})$ Tween 80 , or in $7 \mathrm{H} 9$ broth (Difco) supplemented with $10 \%(\mathrm{v} / \mathrm{v})$ albumin, dextrose and catalase (ADC) (Becton Dickinson), $0.2 \%(\mathrm{v} / \mathrm{v})$ glycerol and $0.05 \%(\mathrm{v} / \mathrm{v})$ Tween 80 , at $37{ }^{\circ} \mathrm{C}$ with shaking at 150 r.p.m. Hygromycin was used at a concentration of $50 \mu \mathrm{g} \mathrm{ml}^{-1}$ and kanamycin at $20 \mu \mathrm{g} \mathrm{m}{ }^{-1}$. Escherichia coli $\mathrm{DH} 5 \alpha$ was grown in Luria-Bertani medium with $100 \mu \mathrm{g}$ ampicillin $\mathrm{ml}^{-1}, 50 \mu \mathrm{g}$ kanamycin $\mathrm{ml}^{-1}$ and $250 \mu \mathrm{g}$ hygromycin $\mathrm{ml}^{-1}$.

\section{Cloning, expression and in vitro disruption of the gInA-1 gene.} The $g \ln A-1$ locus (Mb2244) with upstream promoter sequence was amplified from $M$. bovis genomic DNA by PCR using standard conditions. The amplified DNA was ligated into the pMV261 shuttle vector, producing $\mathrm{pHCl}$, and electroporated into M. smegmatis $\mathrm{mc}^{2}$ 155, as described by Pavelka \& Jacobs (1999). The transformed $M$. smegmatis strain $(M s-g \ln A 1)$ was selected on $7 \mathrm{H} 10$ agar plates containing $25 \mu \mathrm{g}$ kanamycin $\mathrm{ml}^{-1}$ and tested for growth in THP-1 cells.

For in vitro disruption, the $g \ln A-1$ coding sequence was PCRamplified as above. The purified $1.437 \mathrm{~kb}$ product was cloned into the pGEM-T Easy cloning vector, producing plasmid pHC2. The ATPbinding domain of $g \ln A-1$ was deleted by inverse PCR (Pavelka \& Jacobs, 1999) and a KpnI site was created, producing plasmid pHC3. A $1.5 \mathrm{~kb}$ aph cassette with flanking $K p n I$ sites from plasmid pHC4 was excised and ligated into the $g \ln A-1$ coding sequence, producing pHC5.
Generation of the $\boldsymbol{g} \mathbf{I n A - 1}$ mutant of $\boldsymbol{M}$. bovis. A $2.8 \mathrm{~kb} \mathrm{BamHI}$ SpeI fragment from plasmid pHC5 was cloned into the temperaturesensitive vector pPR27 (Pelicic et al., 1997), and the resulting construct (pHC6) was electroporated into M. bovis as described earlier (Pavelka \& Jacobs, 1999). The gentamicin-positive, singlecrossover recombinants were grown to saturation with $10 \mathrm{mM}$ glutamine, and the gentamicin-negative, sucrose-resistant phenotype was selected on 7H10 agar plates containing OADC, $0.2 \%$ Casamino acid, $10 \mathrm{mM}$ glutamine, $2 \%$ sucrose and $20 \mu \mathrm{g}$ kanamycin $\mathrm{ml}^{-1}$. Genomic DNA was isolated from the sucrose-resistant clones and allelic exchange was confirmed by Southern analysis. For complementation, the mutant was electroporated with $\mathrm{pHC7}$ containing the $g \ln A-1$ gene with a promoter in the pNBV1 vector (Howard et al., 1995) and selected on 7H10 plates containing hygromycin.

Phenotypic characterization of the gInA-1 mutant of $M$. bovis. A $50 \mathrm{ml}$ volume of medium was inoculated with 10-day-old primary culture and incubated under shaking conditions at $37^{\circ} \mathrm{C}$. Growth characteristics were studied in $7 \mathrm{H} 9$ medium with enrichment or in Sauton's defined medium with $10 \mathrm{mM}$ glutamine, and optical density was measured periodically. c.f.u. was also determined on supplemented 7H10 agar plates with or without glutamine.

Determination of the GS activity and protein profile of mycobacterial strains. $M$. bovis cells were grown as above, and M. smegmatis was cultured in $7 \mathrm{H} 9$ medium with shaking at $37{ }^{\circ} \mathrm{C}$. Extracellular GS activity was measured by the $\gamma$-glutamyl transfer reaction (Woolfolk et al., 1966). Cultures of $10 \mathrm{ml}$ volume were centrifuged at $8000 \mathrm{~g}$ for $15 \mathrm{~min}$ at $4{ }^{\circ} \mathrm{C}$ and washed once with PBS, and the pellets were resuspended in $5 \mathrm{ml}$ PBS for sonication with a microtip. M. smegmatis was sonicated on ice for five cycles of $30 \mathrm{~s}$ each, and M. bovis for 15 cycles of $30 \mathrm{~s}$ each with intermittent cooling of the same duration, at $35 \%$ amplitude. Cell debris was removed by centrifugation and the clear lysate was filtered through a $0.2 \mu \mathrm{m}$ poresize Millipore filter. The culture filtrates were passed through a $0.22 \mu \mathrm{m}$ pore-size syringe filter and concentrated using a $30 \mathrm{kDa}$ molecular weight cut-off Amicon filter (Millipore). GS activity was expressed as micromoles hydroxamate formed, determined from a standard curve obtained with authentic $\gamma$-glutamyl hydroxamate (Sigma).

The GS protein profile in the cellular and extracellular fractions of $M$. smegmatis strains was determined by SDS-PAGE followed by Western blotting with anti-GS and -GroEL antibodies.

Estimation of PLG in $\boldsymbol{M}$. bovis and $\boldsymbol{M}$. smegmatis strains. The strains were grown in $7 \mathrm{H} 9$ supplemented with $10 \% \mathrm{ADC}, 0.2 \%(\mathrm{v} / \mathrm{v})$ glycerol and $0.05 \%(\mathrm{v} / \mathrm{v})$ Tween 80 with shaking. The cell walls were prepared from 12-15-day-old $M$. bovis or 3-day-old M. smegmatis cultures, and PLG was purified as described elsewhere (Hirschfield et al., 1990). The presence of PLG was confirmed by GC-MS analysis, after hydrolysis of the samples at $110{ }^{\circ} \mathrm{C}$ for $20 \mathrm{~h}$ with $6 \mathrm{M} \mathrm{HCl}$ followed by esterification with heptafluorobutyryl isobutyl anhydride (MacKenzie \& Hogge, 1977). GC-MS was performed using a Shimadzu GC-MS 2010, and an Rtx-5 MS capillary column (Restek), with an oven temperature range of $90-180{ }^{\circ} \mathrm{C}(5 \mathrm{~min})$ at $4{ }^{\circ} \mathrm{C} \min ^{-1}$, raised to $300{ }^{\circ} \mathrm{C}$ at $4{ }^{\circ} \mathrm{C} \mathrm{min}^{-1}$. The injection temperature was $280{ }^{\circ} \mathrm{C}$, with an interface temperature of $290^{\circ} \mathrm{C}$. MS data were matched in the NIST05.LIB and WILEY8.LIB chemical libraries.

\begin{abstract}
Uptake and intracellular growth of $\boldsymbol{M}$. bovis and $\boldsymbol{M}$. smegmatis strains in human THP-1 macrophages. The human THP-1 macrophage monocytic cell line was maintained in RPMI 1640 medium containing $10 \%$ heat-inactivated fetal bovine serum (FBS), $20 \mathrm{mM}$ HEPES and $2 \mathrm{mM}$ L-glutamine at $37{ }^{\circ} \mathrm{C}$ with $5 \% \mathrm{CO}_{2} / 95 \%$ air. Cells were seeded at $2 \times 10^{5}$ cells per well in 24-well tissue-culture plates and differentiated with $20 \mathrm{nM}$ phorbolmyristate acetate (PMA)
\end{abstract}


for $18 \mathrm{~h}$. The efficiency of uptake by the phagocytic cells was determined at increasing bacteria: cell ratios (m.o.i.). For each data point, the mean of triplicate wells was used.

The monolayers were infected with washed, exponential-phase bacteria of $M$. bovis or $M$. smegmatis for $4 \mathrm{~h}$ at m.o.i. values of 5 and 50, respectively. After infection, the cells were treated with medium containing $20 \mu \mathrm{g}$ gentamicin $\mathrm{ml}^{-1}$ for a short time, and washed twice with RPMI medium. Fresh, complete medium was added and the plates were incubated at $37^{\circ} \mathrm{C}$. At different time points the cells were dislodged gently and centrifuged at 2000 r.p.m. for 3 min, washed two times with fresh RPMI medium, and lysed in sterile water. The lysate was diluted in 7H9 medium and plated on 7H11-OADC agar plates with or without $10 \mathrm{mM}$ L-glutamine. The plates were incubated at $37{ }^{\circ} \mathrm{C}$ and colonies were scored after 4 days for M. smegmatis and 3-4 weeks for M. bovis strains. The morphology of the infected cells was observed under a phase-contrast microscope after different time periods, and viability was assessed by trypan blue exclusion (Theus et al., 2004). The data at each time point are the mean from triplicate wells. The results presented are representative of multiple, independent experiments.

Response to mechanical and chemical stress. Twelve to fourteen day-old $M$. bovis cultures with an $\mathrm{OD}_{600}$ ranging from 1.2 to 1.4 $\left(\sim 2 \times 10^{7}\right.$ c.f.u. $\left.\mathrm{ml}^{-1}\right)$ were subjected to mechanical stress by sonication with seven cycles of $30 \mathrm{~s}$ duration at $25 \%$ amplitude, with intermittent cooling of the same duration. In another experiment, the bacilli were treated with lysozyme at a concentration of $1 \mathrm{mg} \mathrm{ml}^{-1}$ for $24 \mathrm{~h}$ and c.f.u. was determined for the treated and untreated samples by plating as described above.

Biofilm formation. For biofilm formation, $500 \mu$ l Sauton's medium in 24-well polystyrene tissue-culture plates was inoculated with $5 \mu \mathrm{l}$ planktonic cultures (30 days old, $\mathrm{OD}_{600}$ normalized to 1) and incubated for 3-4 weeks. Biofilm was quantified by removing the medium carefully and staining with $1 \%$ crystal violet for $45 \mathrm{~min}$. The wells were washed three times with water and air-dried. The dye was solubilized with $80 \%$ ethanol and $A_{550}$ was measured (O'Toole et al., 1999).

SDS sensitivity test. $M$. bovis strains were grown to exponential phase (15 days; $\mathrm{OD}_{600}$ ranging from 1.2 to 1.4 ), and $6 \mu$ of 10 -fold diluted culture was spotted onto $7 \mathrm{H} 10$ agar plates containing $10 \%$ OADC, $0.05 \%$ Tween 80 and $0.01 \%$ SDS. Plates were incubated at $37{ }^{\circ} \mathrm{C}$ for 3-4 weeks and c.f.u. was recorded.

Drug sensitivity test. Drug sensitivity was tested by the resazurin microplate assay (REMA) (Palomino et al., 2002) or by the agar dilution method (Hanson \& Martin, 1978). Briefly, the drugs were prepared in either DMSO or sterile deionized water, and twofold serial dilutions were made in $7 \mathrm{H} 9$ growth medium in microtitre plates. The drug concentrations ranged from 0.003 to $1 \mu \mathrm{g} \mathrm{ml}^{-1}$ for isoniazid, 0.0075 to $2 \mu \mathrm{g} \mathrm{ml}^{-1}$ for ethambutol, 0.00007 to $0.018 \mu \mathrm{g}$ $\mathrm{ml}^{-1}$ for rifampicin, and 0.39 to $100 \mu \mathrm{g} \mathrm{ml} \mathrm{m}^{-1}$ for D-cycloserine. Approximately $5 \times 10^{4}$ c.f.u. of a 12 -day-old $M$. bovis culture was added per well; control wells contained only bacteria and growth medium. The plates were incubated at $37{ }^{\circ} \mathrm{C}$ for 7 days and $0.02 \%$ resazurin was added. The plates were observed after $48 \mathrm{~h}$ for a colour change from blue to pink. The visual MIC was determined as the lowest concentration of the drug at which a colour change was prevented. In the agar dilution method, $10 \mu \mathrm{l}$ culture was spotted onto an agar plate containing the drug and incubated at $37{ }^{\circ} \mathrm{C}$ for $3-4$ weeks. The MIC was recorded as the concentration at which bacterial growth was completely inhibited.

Virulence in BALB/c mice. Six-week-old male BALB/c mice (18$20 \mathrm{~g}$ ) were challenged intravenously through the lateral tail vein with exponential-phase cultures of M. bovis strains. Animals were weighed twice monthly and checked daily for clinical symptoms of weight loss and lack of response to stimuli, as displayed in rodent tuberculosis. The mice $(n=3)$ from each group were sacrificed at 2, 4, 6 and 15 weeks, and the lungs and spleens were cultured individually for c.f.u. determination on $7 \mathrm{H} 11$ plates with or without $10 \mathrm{mM}$ L-glutamine. Colonies were scored after $3-4$ weeks incubation at $37^{\circ} \mathrm{C}$. Animal research was conducted in compliance with animal ethics committee and tuberculosis challenge facility guidelines.

\section{RESULTS}

\section{Construction and characterization of the $M$. bovis gInA-1 mutant}

Supplementary Fig. S1(a) shows a schematic map of the insertional inactivation of the $g \ln A-1$ locus. Genomic DNA from two sucrose- and kanamycin-resistant colonies reacted with the labelled probe in a Southern blot after SmaI digestion. A $\sim 3.8 \mathrm{~kb}$ band was observed in the mutant as compared with a $2.3 \mathrm{~kb}$ fragment in the wildtype (Supplementary Fig. S1b), indicating allelic exchange of the wild-type gene by the disrupted copy; colony 1 was used for further characterization.

\section{Growth characteristics}

Growth of the mutant in 7H9 broth with enrichment showed a longer lag phase than the wild-type strain (Fig. 1a, c), but later cell densities and c.f.u. values were not very different in the two strains, indicating that the $g \ln A-1$ strain was able to acquire nitrogen from other sources in the medium [L-glutamic acid, $3.4 \mathrm{mM}$; ammonium sulphate, $3.8 \mathrm{mM}$; ferric ammonium citrate, $0.14 \mathrm{mM}$; and enrichment containing $10 \%(\mathrm{v} / \mathrm{v})$ albumin]. In contrast to $M$. tuberculosis, the $M$. bovis glnA-1 mutant replicated for 3-4 logs in the rich medium with no L-glutamine addition. However, in Sauton's defined medium, no growth was observed in the absence of glutamine. The growth defect was abolished in the presence of $10 \mathrm{mM}$ glutamine (data not shown).

\section{GS activity and PLG content in the cell wall}

Under aerobic conditions, the GS activity peaked on day 10 in M. bovis strains. The wild-type contained twofold higher intracellular GS activity compared with the mutant (Fig. $1 \mathrm{~b})$; the activity was restored to the wild-type level in the complemented strain, indicating the direct involvement of the $g \ln A-1$ gene in total cellular GS activity. The extracellular activity was proportionately reduced in both strains: the wild-type had about one-sixth of the intracellular activity, while the mutant showed only a basal level of activity which was similar to that of the medium alone (Fig. 1d). Like the enzyme activity, the PLG content of the cell wall was also drastically reduced in the mutant (below detectable limits) compared with $\sim 4$ and $5 \%$ of the dry weight of the cell wall in the wild-type and complemented strains, respectively. 

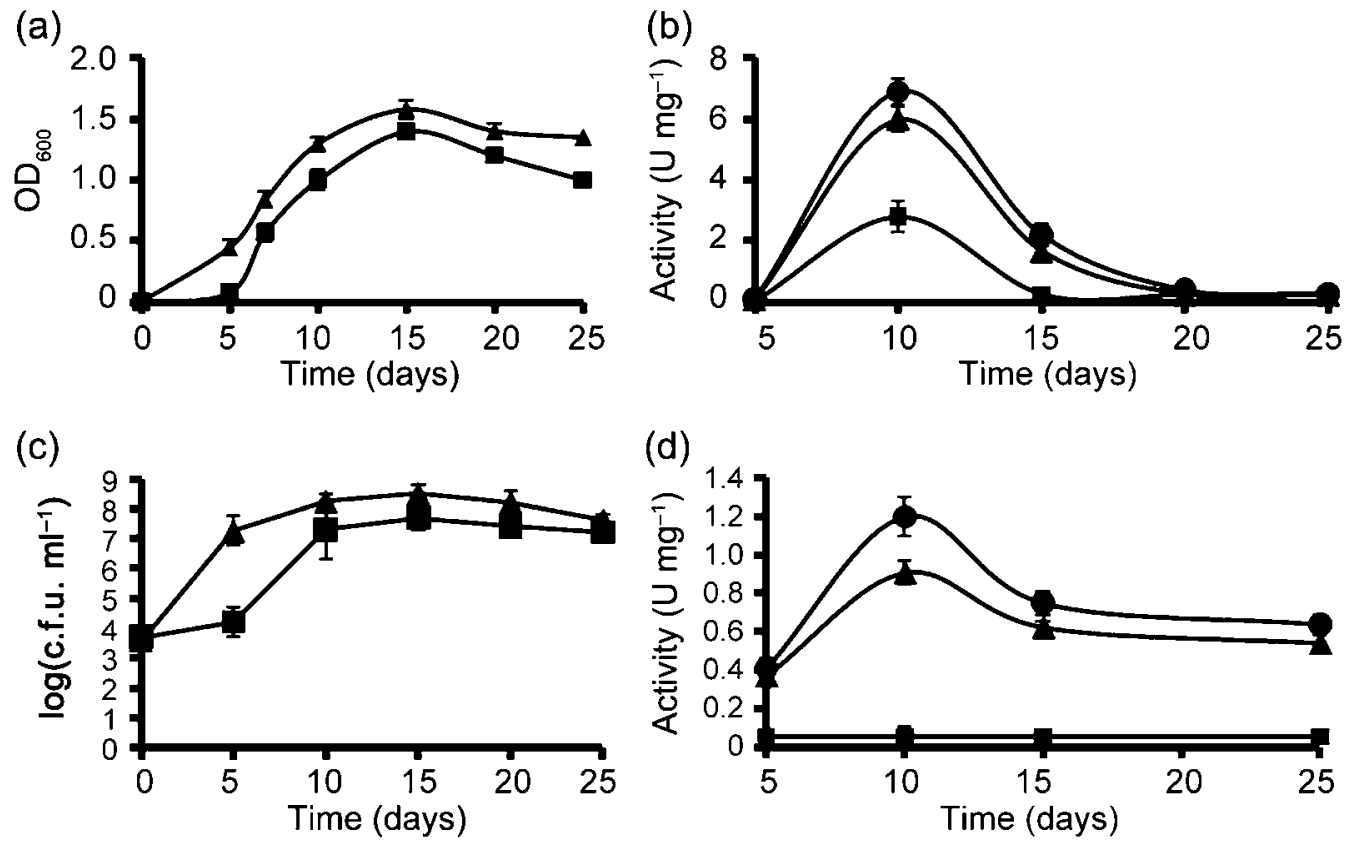

Fig. 1. Growth and enzyme activity of the $g \ln A-1$ mutant in broth culture. (a) The glnA-1 mutant ( $\boldsymbol{\square}$ ) and wild-type M. bovis ( $\mathbf{\Delta}$ ) were inoculated to an initial optical density of 0.004-0.005 in enriched 7H9 medium. (a) $\mathrm{OD}_{600}$, (c) c.f.u., (b and d) intra- and extracellular GS activity, respectively. $g \ln A-1$ mutant $(\boldsymbol{\square})$, wild-type $M$. bovis $(\mathbf{\Delta})$ and complemented $g \ln A-1(\boldsymbol{\bullet})$. Data are mean $\pm S D$ of values obtained from three independent cultures.

Control M. smegmatis cells (containing pMV261) produced a very low level of endogenous GS protein compared with cells expressing the $g \ln A-1$ gene episomally (Fig. 2a, b). Most of the catalytically active GS (85\%) was released extracellularly by $M s-g \ln A 1$, in contrast to wild-type $M$. bovis, in which only $\sim 14 \%$ of the endogenous GS was detected in the culture supernatant (Fig. 2a). The GS protein was detected in the culture medium of the $\mathrm{Ms}$ -
glnA1 strain after $24 \mathrm{~h}$, probably exported by a specific mechanism and not by cell lysis, as an intracellular protein, GroEL, though produced in the cell, was not detected (Fig. 2b). The PLG content of $M s-g \ln A 1$ was found to be 10 $12 \%$ of the cell wall dry weight, which was more than twice the amount obtained from native M. bovis and could be due to expression from a multicopy plasmid. The control vector-transformed cells produced no PLG. These results

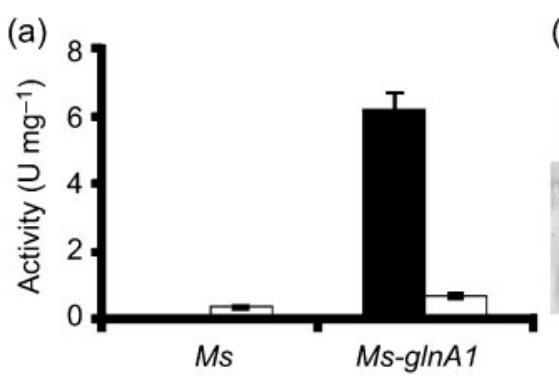

(b)

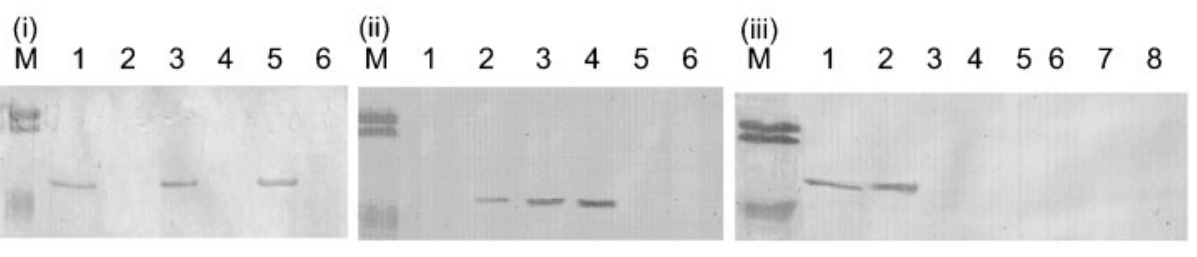

Fig. 2. Protein expression and GS activity in $M$. smegmatis strains. (a) Enzyme activity in the cell lysate and culture supernatant fractions at 48 h. (b) Western blots of the fractions analysed by anti-GS antibodies. M, prestained protein markers. (i) Lanes: 1 , 3 and 5, cell lysate of $M s-g \ln A 1 ; 2,4$ and 6, wild-type. (ii) Lanes: 1-4, culture supernatant of Ms-glnA1 at 15, 24, 48 and 56 h, respectively; 5 and 6, wild-type strain at 48 and 56 h, respectively. (iii) Blotting with anti-GroEL antibodies: lanes 1 and 2, cell lysates of $M s-g \ln A 1$ and wild-type, respectively, at $48 \mathrm{~h}$; lanes 3, 4 and 5, culture supernatants of $M s-g \ln A 1$ at 24,48 and $56 \mathrm{~h}$, respectively; lanes 6,7 and 8 , wild-type at 24,48 and 56 h respectively. 
demonstrate the involvement of the $g \ln A-1$ gene in cell wall-associated PLG synthesis.

\section{Intracellular growth of $\boldsymbol{M}$. bovis and $\boldsymbol{M}$. smegmatis strains in human THP-1 cells}

To examine whether the altered cell wall chemistry played a role in the uptake of the mutant by THP-1 macrophages, the m.o.i. values of the mutant and the wild-type were compared. To achieve internalization of an average of two to six bacteria per macrophage, a higher m.o.i. was required for the mutant than for the wild-type (Fig. 3a), suggesting alterations in the cell surface. When cells were infected with wild-type $M$. bovis, the viability of the cells was reduced to $51 \%$ in $48 \mathrm{~h}$, as determined by trypan blue exclusion; the $g \ln A-1$ mutant caused less damage to the cells $(81 \%$ viability), reflecting the reduced virulence of the strain. The complemented strain restored the wild-type phenotype, reducing cellular viability to $49 \%$ (Fig. 3b).

The mutant showed an attenuated phenotype in macrophages cultured in standard RPMI medium $(2 \mathrm{mM}$ glutamine), showing a reduction of more than $1 \log$ in
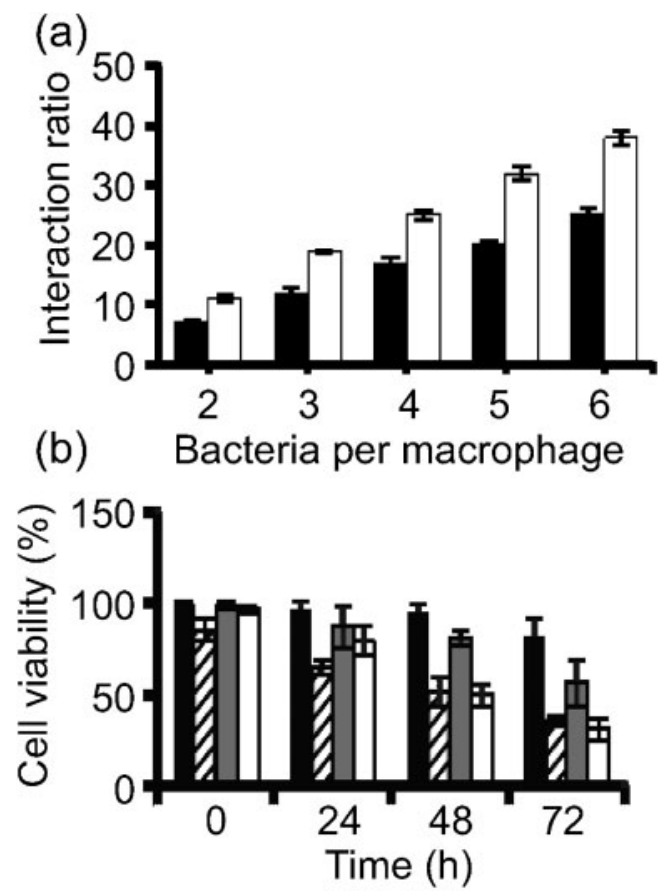

Fig. 3. Uptake of $M$. bovis strains and viability of infected THP-1 cells. (a) Macrophages were infected and the number of internalized bacteria was determined by plating at time zero. Black bars, wild-type $M$. bovis; white bars, $g \ln A-1$ mutant. (b) Cellular viability by trypan blue exclusion. Black bars, control uninfected cells; hatched bars, wild-type $M$. bovis; grey bars, glnA1 mutant; white bars, $g \ln A-1^{+}$complemented mutant. Data are mean \pm SEM of triplicate wells and are representative of three independent experiments. (a)
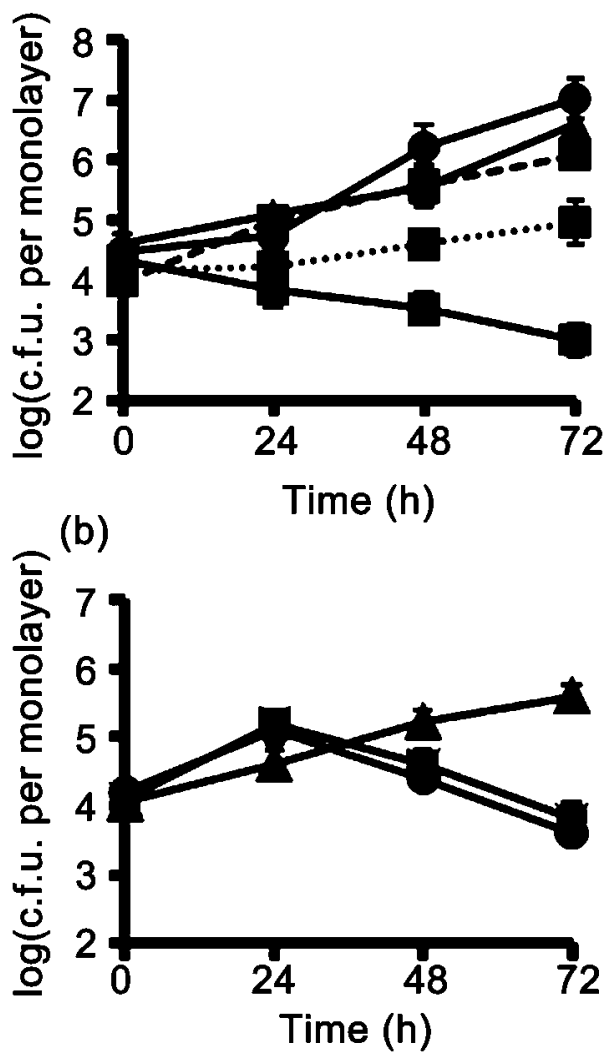

Fig. 4. Intracellular growth of $M$. bovis and $M$. smegmatis strains in THP-1 cells. Cells were cultured in RPMI medium containing $2 \mathrm{mM}$ L-glutamine. (a) Solid lines: (A) wild-type, (0) $g \ln A^{+}$ complemented, ( $\mathbf{\square})$ glnA-1 mutant. The mutant was also grown in cells cultured in RPMI with $5 \mathrm{mM}$ (dotted line) and $10 \mathrm{mM}$ (dashed line) L-glutamine. (b) (o) M. smegmatis, (ם) M. smegmatis containing vector pMV261, (A) Ms-glnA1. The data presented are mean \pm SEM of triplicate wells and are representative of three individual experiments.

c.f.u. after $72 \mathrm{~h}$, whereas the wild-type strain multiplied, showing an increase of more than 2 logs after $72 \mathrm{~h}$. Complementation of the mutant with $g \ln A-1$ in a plasmid restored the growth defect of the mutant (Fig. 4a). When the tissue-culture medium was supplemented with 5 and $10 \mathrm{mM}$ glutamine, the c.f.u. of the mutant strain increased by $\sim 1$ and 2 logs, respectively, at $72 \mathrm{~h}$, indicating its dependence on extracellular glutamine in vivo (Fig. 4a). A higher glutamine concentration of $20 \mathrm{mM}$ was inhibitory to the bacteria (data not shown).

$M s$-glnA1 survived in the THP-1 cells longer, to $72 \mathrm{~h}$ $(P<0.001)$, than the untransformed or vector-transformed control cells (Fig. $4 \mathrm{~b}$ ). In the control group, an increase of $\sim 1 \log$ occurred at $24 \mathrm{~h}$, after which the number of c.f.u. declined. In contrast, M. smegmatis cells containing heterologously expressed $g \ln A-1$ continued to replicate up to $72 \mathrm{~h}$ within the macrophages, showing the necessity of GS for intracellular survival. 


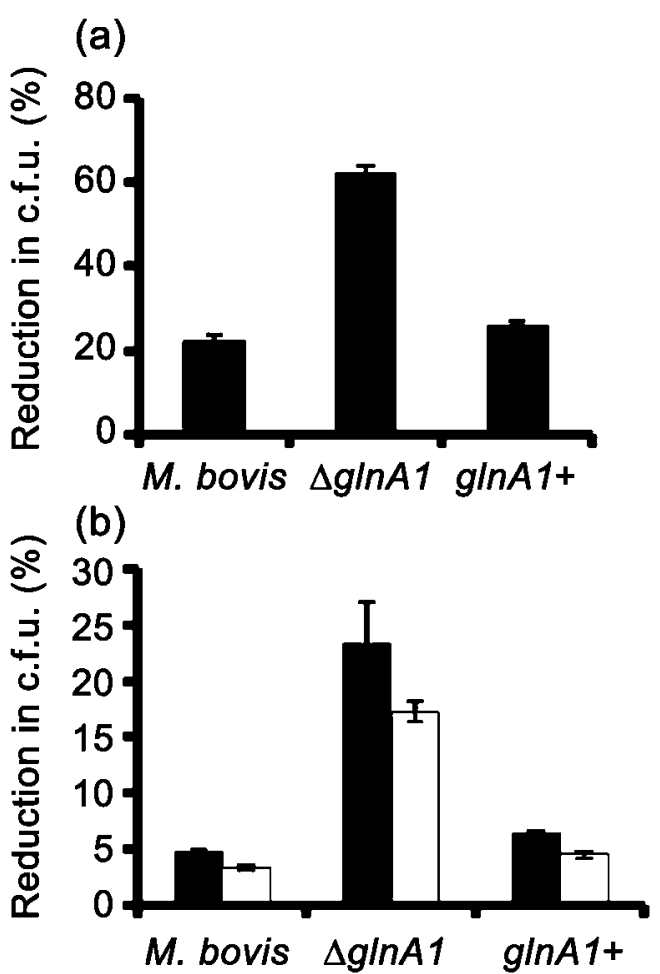

Fig. 5. Sonication and lysozyme sensitivity of the $g \ln A-1$ mutant. (a) Cells were treated with lysozyme at a concentration of $1 \mathrm{mg}$ $\mathrm{ml}^{-1}$ for $24 \mathrm{~h}$ and plated for c.f.u. determination. (b) Cultures were sonicated and plated; black bars, 12-day-old cultures; white bars, 25-day-old cultures. Error bars, SD from three independent experiments.

\section{Cell wall sensitivity of the $g \ln A-1$ mutant}

The cell wall of the mutant was highly suceptible to physical and chemical stresses. Sonication of a 12-15-day-old culture resulted in a $62 \%$ reduction in c.f.u. of the mutant in comparison with a $22 \%$ reduction in the wild-type strain, corresponding to an approximately threefold increase in the sensitivity of the mutant (Fig. 5a). At a later time point (30 days), the mutant cells became somewhat more resistant to sonication (data not shown). The complemented strain behaved similarly to the wild-type. Likewise, the mutant was more sensitive to lysozyme treatment, showing a four- to fivefold increase in sensitivity compared with the wild-type or the complemented strain, in both the exponential and stationary phases of growth (Fig. 5b). The SDS tolerance of the mutant was also reduced drastically compared with the wild-type strain, and the defect was abolished in the complemented strain, reflecting alterations in the chemistry of the cell wall (Fig. 6).

\section{Effect on biofilm formation}

The mutant was defective in forming biofilms on a polystyrene surface. The mutant cells showed an $\sim 50 \%$ reduction in biofilm formation, while complementation restored the defect to a large extent, reiterating the involvement of $g \ln A-1$ in modulating the cell surface properties of mycobacteria (Fig. 7).

\section{Drug suceptibility test}

MIC values for various drugs are shown in Table 1. In the $\operatorname{gn} A-1$ strain, the MIC values for rifampicin and Dcycloserine were reduced about eightfold compared with the wild-type strain. The MIC values for ethambutol and isoniazid remained unchanged in the mutant; however, in the $g \ln A-1$ complemented strain, the two drugs had twoand fourfold higher MICs than the wild-type strain, which could be due to expression of the protein in higher copy number from the plasmid, causing a higher deposition of PLG in the cell wall.
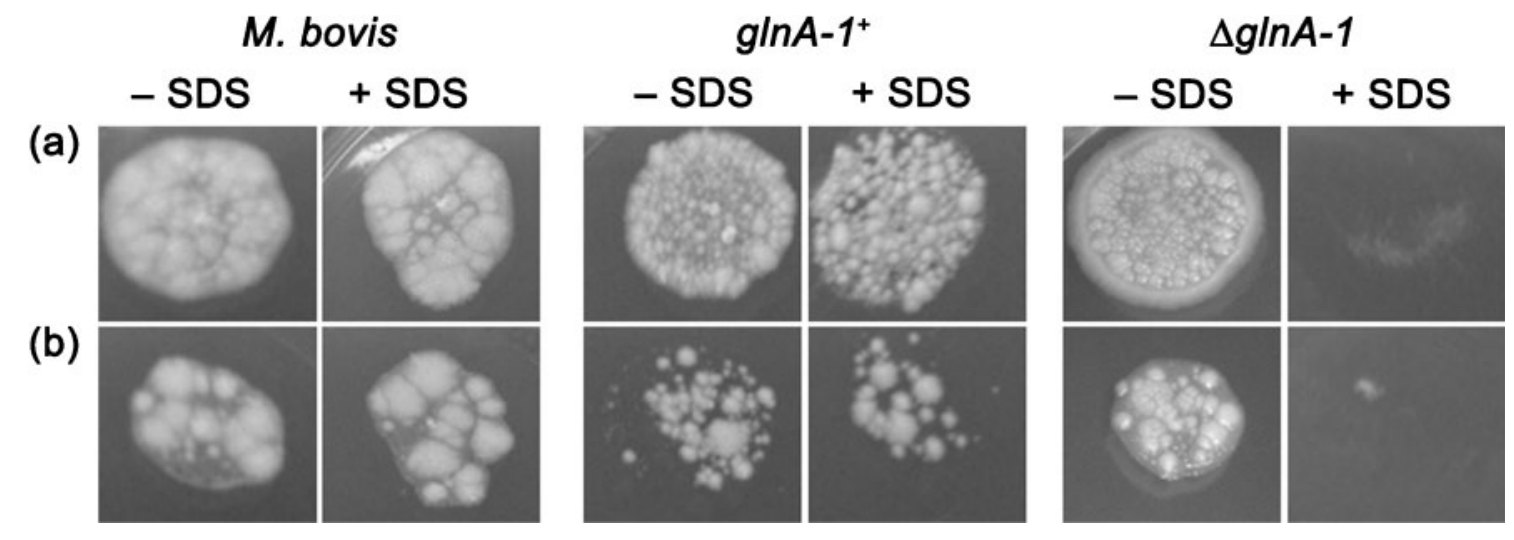

Fig. 6. SDS sensitivity of the $g \ln A-1$ mutant. Dilutions of 12 -day-old bacterial cultures were spotted onto $7 \mathrm{H} 10$ agar plates with (+SDS) or without (-SDS) $0.01 \%$ SDS and incubated at $37{ }^{\circ} \mathrm{C}$ for 3-4 weeks. (a) Undiluted culture, (b) $1: 10$ diluted culture. 


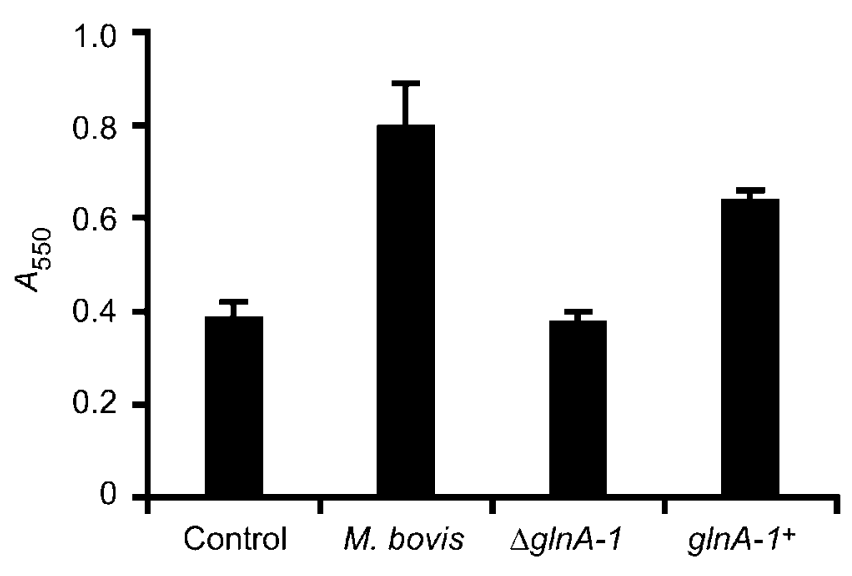

Fig. 7. Biofilm formation by $M$. bovis strains. Stationary phase planktonic cultures were inoculated in Sauton's medium containing $10 \mathrm{mM}$ L-glutamine and incubated for 4 weeks. Adherent biofilm was stained with $1 \%$ crystal violet and $A_{550}$ was measured. Error bars, SEM from triplicate wells. The experiment was repeated three times with similar results. Control, medium only.

\section{Replication in BALB/c mice}

The ability of $M$. bovis strains to survive and replicate in the spleen and lungs of $\mathrm{BALB} / \mathrm{c}$ mice was followed for up to 15 weeks. The c.f.u. counts on day 0 of the three cultures were wild-type $1 \times 10^{5}, \operatorname{gln} A-1$ mutant $1.7 \times 10^{5}$ and complemented strain $1.4 \times 10^{5}$. At 2 weeks post-injection, the number of c.f.u. dropped slightly in all the groups. After 4 weeks, the number of mutant cells was reduced to $\sim 10^{2}$ in the organs (Fig. 8a), and at 6 weeks no colonies were obtained (Fig. 8b). In contrast, both the wild-type and complemented strains infected lungs and spleen, and after an initial fall, the bacterial burden increased by $2-3$ logs at 15 weeks (Fig. 8c). No deaths were recorded until 19 weeks in any group.

Table 1. MIC values of antitubercular drugs for M. bovis strains, determined by REMA

Each concentration of drug was tested in triplicate. The data were obtained from three independent experiments and similar results were obtained each time.

\begin{tabular}{|c|c|c|c|}
\hline \multirow[t]{2}{*}{ Drug } & \multicolumn{3}{|c|}{$\operatorname{MIC}^{\star}\left(\mu \mathrm{g} \mathrm{ml}^{-1}\right)$} \\
\hline & M. bovis & $g \ln A-1$ mutant & $g \ln A-1^{+}$ \\
\hline Isoniazid & 0.06 & 0.06 & 0.25 \\
\hline Ethambutol & 0.5 & 0.5 & 1 \\
\hline Rifampicin & 0.002 & 0.00031 & 0.002 \\
\hline D-Cycloserine & 25 & 6.25 & 25 \\
\hline
\end{tabular}

${ }^{*}$ MIC was defined as the lowest concentration of the drug that prevented a change in colour from blue to pink.
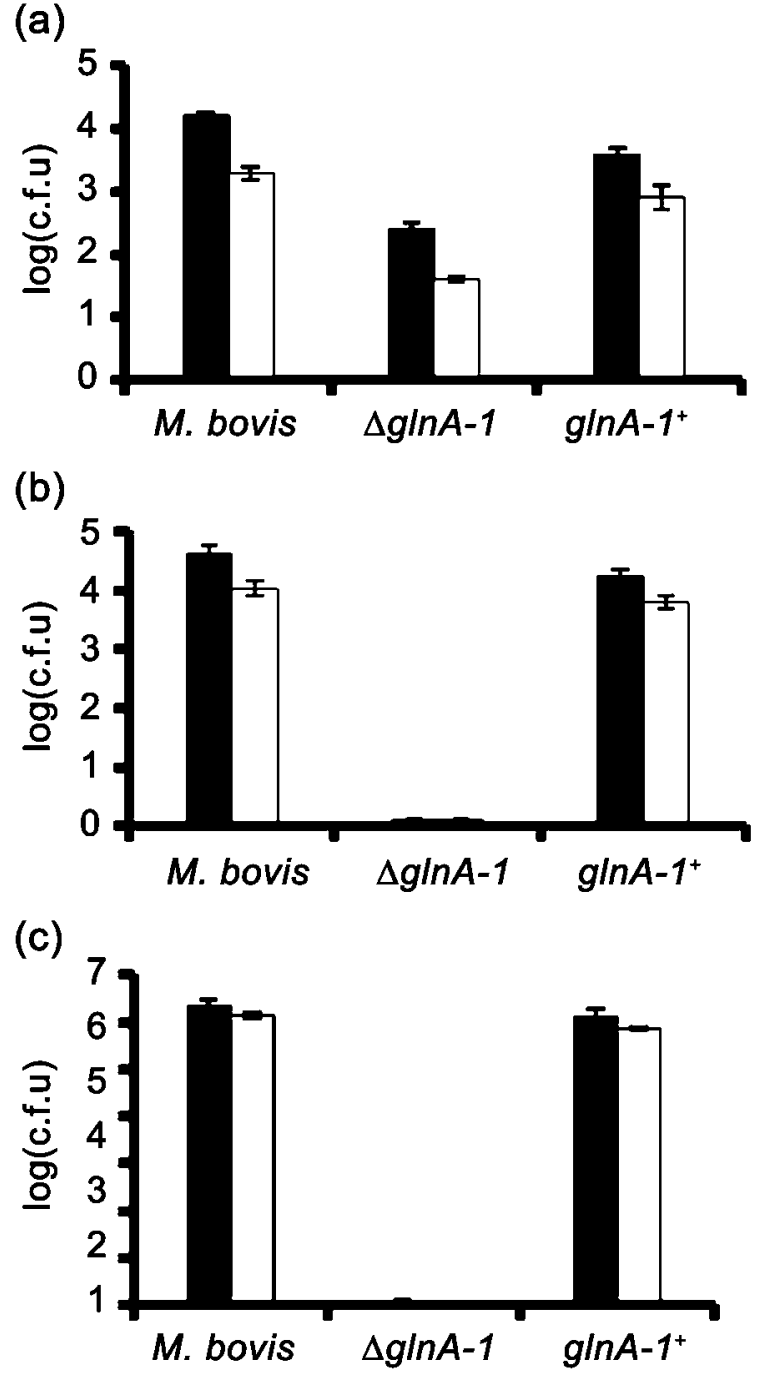

Fig. 8. Growth of $M$. bovis strains in BALB/c mice. Each group containing three mice was infected through the lateral tail vein with a dose of $1-2 \times 10^{5}$ c.f.u. of each $M$. bovis strain. c.f.u values are shown at (a) 4 weeks, (b) 6 weeks and (c) 15 weeks. Lungs and spleens from each mouse were homogenized and dilutions were plated. Black bars, lungs; white bars, spleen. Data are the mean \pm SD from three individual mice, and are representative of two independent experiments.

\section{DISCUSSION}

The inherent resistance of mycobacteria to common antibiotics and stressful conditions in the host is largely attributed to the unique chemistry of their cell walls (Hett \& Rubin, 2008; Vandal et al., 2009). Several earlier studies have reported the presence of poly-L-glutamate/PLG in the cell wall of pathogenic strains (Wietzerbin et al., 1975; Hirschfield et al., 1990; Harth \& Horwitz, 1999); however, very little is known about its physiological role. Since PLG is associated with the cell wall, it is likely to influence the cell envelope chemistry and consequently the response of the pathogen to various stress factors. 
The association of PLG exclusively with pathogenic species emphasizes its importance in mycobacterial pathogenesis. Other pathogenic Gram-positive bacilli, such as Bacillus anthracis and Staphylococcus epidermidis, produce a poly- $\gamma$ glutamate capsule on the cell surface as a virulence factor (Candela \& Fouet, 2006). Apparently, the loss of PLG decreases the mechanical strength of the $M$. bovis cell wall and increases its sensitivity to an important effector of innate immunity, lysozyme, resulting in faster clearance of the mutant from THP-1 cells. In view of the greater resistance of mycobacterial peptidoglycan (PG) to lysozyme (Hett \& Rubin, 2008), the higher sensitivity of the $g \ln A-1$ mutant to the latter demonstrates the participation of PLG in protecting the murein layer, either by reducing its accessibility to the enzyme or by chemically fortifying the PG sacculus against enzyme attack. Further, the increased sensitivity of the $g \ln A-1$ mutant to SDS also supports the role of the SDS-insoluble PLG in cell wall protection.

The defect in biofilm formation could be due to the changed surface chemistry of the mutant. The ability to form biofilms is an important virulence determinant of pathogenic mycobacteria (Ojha et al., 2008). The biofilm formed by $M$. tuberculosis is known to contain abundant free mycolic acid (Ojha et al., 2005, 2008); at this stage we do not know whether mycolic acid synthesis or its content in the cell wall of the mutant is affected. Other genetic loci identified in biofilm formation in mycobacteria are linked to enzymes of the tricarboxylic acid (TCA) cycle, glycolipid biosynthesis (Yamazaki et al., 2006) and extracellular GroEL1 (Ojha et al., 2005). Moreover, how GS influences biofilm formation in $M$. bovis needs further investigation.

The greater susceptibility of the mutant to the lipophilic antitubercular drugs rifampicin and D-cycloserine was expected, and was more likely due to an increased permeability of the cell wall and not a direct effect on the targets, the $\beta$-subunit of RNA polymerase and D-Ala-D-Ala ligase, respectively. This also explains the unchanged MICs observed for the non-lipophilic antibiotics isoniazid and ethambutol, which also act on cell wall targets.

The physiological role of $g \ln A-1$ in sustaining bacterial growth and survival within the host cells is reiterated by the increased mean survival time of the $M s-g \ln A 1$ strain in THP-1 cells. It is intriguing that M. smegmatis, despite four homologues $(g \ln A 1-4)$ in the genome, does not produce a GS protein, and that the presence of the M. bovis $g \ln A-1$ locus leads to synthesis of PLG in $M$. smegmatis. A comparative analysis of the two loci may reveal interesting information about the gene expression of pathogenic mycobacteria. It has also been postulated that GS, by assimilation of ammonium ions into amino acids as glutamate and glutamine (Harper et al., 2008; Amon et al., 2009; Merrick \& Edwards, 1995), helps in the removal of excess, toxic ammonia from the intraphagosomal milieu, thereby promoting the survival of the pathogen; however, this possibility needs to be examined experimentally. Based on our results, it may be argued that in addition to alterations in bacterial metabolism, the chemical changes brought about in the cell wall due to the absence of PLG play a significant role in the attenuation of M. bovis.

The diverse phenotypic changes caused by disruption of the $\operatorname{gn} A-1$ gene provide a basis for using it as a drug target as well as a vaccine candidate. However, the utility of GS as a drug target is somewhat limited due to its similarity to the mammalian enzyme in the relative placement and orientation of all the substrates and cofactors, except the nucleotide-binding site (Krajewski et al., 2008). The pathway of PLG synthesis in mycobacteria is not known; whether it is synthesized intracellularly and carried to the surface or assembled extracellularly, the enzymes involved and the specific role(s) of $g \ln A-1$ need to be established to identify potential new drug targets against tuberculosis.

In conclusion, although the roles of multiple GSs of mycobacteria have been investigated extensively in the past, surprisingly, none of the studies was focused on the impact of PLG depletion from the cell wall. Our data for the first time reveal the importance of this cell wall polymer in enhancing the stress tolerance of the pathogen and open new possibilities for the development of antitubercular drugs.

\section{ACKNOWLEDGEMENTS}

This work was supported by the Department of Biotechnology (DBT), India. We are grateful to Dr V. Pelicic, Imperial College London, UK, for providing the allelic exchange vector pPR27 and Dr M. A. Horwitz, UCLA School of Medicine, Los Angeles, CA, USA, for the plasmid DNA pNBV1. GC-MS analysis was done at the Advanced Instrumentation Facility, Jawaharlal Nehru University. All the animal experiments and Mycobacterium culture work were conducted at the Tuberculosis Aerosol Challenge Facility (TACF) laboratory, International Centre for Genetic Engineering and Biotechnology.

\section{REFERENCES}

Amon, J., Titgemeyer, F. \& Burkovski, A. (2009). A genomic view on nitrogen metabolism and nitrogen control in mycobacteria. $J \mathrm{Mol}$ Microbiol Biotechnol 17, 20-29.

Candela, T. \& Fouet, A. (2006). Poly-gamma-glutamate in bacteria. Mol Microbiol 60, 1091-1098.

Cole, S. T., Brosch, R., Parkhill, J., Garnier, T., Churcher, C., Harris, D., Gordon, S. V., Eiglmeier, K., Gas, S. \& other authors (1998). Deciphering the biology of Mycobacterium tuberculosis from the complete genome sequence. Nature 393, 537-544.

Fisher, S. H. (1999). Regulation of nitrogen metabolism in Bacillus subtilis: vive la difference! Mol Microbiol 32, 223-232.

Flynn, J. L. \& Chan, J. (2001). Tuberculosis: latency and reactivation. Infect Immun 69, 4195-4201.

Garnier, T., Eiglmeier, K., Camus, J. C., Medina, N., Mansoor, H., Pryor, M., Duthoy, S., Grondin, S., Lacroix, C. \& other authors (2003). The complete genome sequence of Mycobacterium bovis. Proc Natl Acad Sci U S A 100, 7877-7882. 
Hanson, C. W. \& Martin, W. J. (1978). Modified agar dilution method for rapid antibiotic susceptibility testing of anaerobic bacteria. Antimicrob Agents Chemother 13, 383-388.

Harper, C., Hayward, D., Wild, I. \& Helden, P. V. (2008). Regulation of nitrogen metabolism in Mycobacterium tuberculosis: a comparision with mechanisms in Corynebacterium glutamicum and Streptomyces coelicolor. IUBMB Life 60, 643-650.

Harth, G. \& Horwitz, M. A. (1999). An inhibitor of exported Mycobacterium tuberculosis glutamine synthetase selectively blocks the growth of pathogenic Mycobacterium in axenic culture and in human monocytes: extracellular proteins as potential novel drug targets. J Exp Med 189, 1425-1436.

Harth, G., Clemens, D. L. \& Horwitz, M. A. (1994). Glutamine synthetase of Mycobacterium tuberculosis: extracellular release and characterization of its enzymatic activity. Proc Natl Acad Sci U S A 91, 9342-9346.

Harth, G., Zamecnik, P. C., Tang, J. Y., Tabatadze, D. \& Horwitz, M. A. (2000). Treatment of Mycobacterium tuberculosis with antisense oligonucleotides to glutamine synthetase mRNA inhibits glutamine synthetase activity, formation of the poly-L-glutamate/glutamine cell wall structure, and bacterial replication. Proc Natl Acad Sci U S A 97, 418-423.

Harth, G., Masleša-Galić, S., Tullius, M. V. \& Horwitz, M. A. (2005). All four Mycobacterium tuberculosis glnA genes encode glutamine synthetase activities but only GlnAl is abundantly expressed and essential for bacterial homeostasis. Mol Microbiol 58, 1157-1172.

Hett, E. C. \& Rubin, E. J. (2008). Bacterial growth and cell division: a mycobacterial perspective. Microbiol Mol Biol Rev 72, 126-156.

Hirschfield, G. R., McNeil, M. \& Brennan, P. J. (1990). Peptidoglycanassociated polypeptides of Mycobacterium tuberculosis. J Bacteriol 172, 1005-1013.

Howard, N. S., Gomez, J. E., Ko, C. \& Bishai, W. R. (1995). Color selection with a hygromycin-resistance-based Escherchia coli-mycobacterial shuttle vector. Gene 166, 181-182.

Krajewski, W. W., Collins, R., Holmberg-Schiavone, L., Jones, A., Karlberg, T. \& Mowbray, S. L. (2008). Crystal structures of mammalian glutamine synthetases illustrate substrate-induced conformational changes and provide opportunities for drug and herbicide design. J Mol Biol 375, 217-228.

MacKenzie, S. L. \& Hogge, L. R. (1977). Gas chromatography mass spectrometry of the $\mathrm{N}(\mathrm{O})$-heptafluorobutyryl isobutyl esters of the protein amino acids using electron impact ionization. J Chromatogr 132, 485-493.

Merrick, M. J. \& Edwards, R. A. (1995). Nitrogen control in bacteria. Microbiol Rev 59, 604-622.

Ojha, A., Anand, M., Bhatt, A., Kremer, L., Jacobs, W. R., Jr \& Hatfull, G. F. (2005). GroEL1: a dedicated chaperone involved in mycolic acid biosynthesis during biofilm formation in mycobacteria. Cell 123, 861-873.

Ojha, A. K., Baughn, A. D., Sambandan, D., Hsu, T., Trivelli, X., Guerardel, Y., Alahari, A., Kremer, L., Jacobs, W. R., Jr \& Hatfull, G. F.
(2008). Growth of Mycobacterium tuberculosis biofilms containing free mycolic acids and harbouring drug-tolerant bacteria. Mol Microbiol 69, 164-174.

O’Toole, G. A., Pratt, L. A., Watnick, P. I., Newman, D. K., Weaver, V. B. \& Kolter, R. (1999). Genetic approaches to study of biofilms. Methods Enzymol 310, 91-109.

Palomino, J. C., Martin, A., Camacho, M., Guerra, H., Swings, J. \& Portaels, F. (2002). Resazurin microtiter assay plate: simple and inexpensive method for detection of drug resistance in Mycobacterium tuberculosis. Antimicrob Agents Chemother 46, 2720-2722.

Pavelka, M. S., Jr \& Jacobs, W. R., Jr (1999). Comparison of the construction of unmarked deletion mutations in Mycobacterium smegmatis, Mycobacterium bovis Bacillus Calmette-Guérin, and Mycobacterium tuberculosis $\mathrm{H} 37 \mathrm{Rv}$ by allelic exchange. J Bacteriol 181, 4780-4789.

Pelicic, V., Jackson, M., Reyrat, J. M., Jacobs, W. R., Jr, Gicquel, B. \& Guilhot, C. (1997). Efficient allelic exchange and transposon mutagenesis in Mycobacterium tuberculosis. Proc Natl Acad Sci U S A 94, 10955-10960.

Reitzer, L. J. (1996). Ammonia assimilation and the biosynthesis of glutamine, glutamate, aspartate, L-alanine, and D-alanine. In Escherichia coli and Salmonella typhimurium, 2nd edn, pp. 391-407. Edited by F. C. Neidhardt \& R. Curtiss. Washington, DC: American Society for Microbiology.

Theus, S. A., Cave, M. D. \& Eisenach, K. D. (2004). Activated THP-1 cells: an attractive model for the assessment of intracellular growth rates of Mycobacterium tuberculosis isolates. Infect Immun 72, 11691173.

Tullius, M. V., Harth, G. \& Horwitz, M. A. (2003). Glutamine synthetase GlnAl is essential for growth of Mycobacterium tuberculosis in human THP-1 macrophages and guinea pigs. Infect Immun 71, 39273936.

Vandal, O. H., Roberts, J. A., Odaira, T., Schnappinger, D., Nathan, C. F. \& Ehrt, S. (2009). Acid-susceptible mutants of Mycobacterium tuberculosis share hypersusceptibility to cell wall and oxidative stress and to the host environment. J Bacteriol 191, 625-631.

Wietzerbin, J., Lederer, F. \& Petit, J. F. (1975). Structural study of the poly-L-glutamic acid of the cell wall of Mycobacterium tuberculosis var hominis, strain Brevannes. Biochem Biophys Res Commun 62, 246252.

Woolfolk, C. A., Shapiro, B. \& Stadtman, E. R. (1966). Regulation of glutamine synthetase. I. Purification and properties of glutamine synthetase from Escherichia coli. Arch Biochem Biophys 116, 177192.

Yamazaki, Y., Danelishvili, L., Wu, M., MacNab, M. \& Bermudez, L. E. (2006). Mycobacterium avium genes associated with the ability to form a biofilm. Appl Environ Microbiol 72, 819-825.

Edited by: M. Daffé 